\title{
An ADMM-based Regularized State Estimation Scheme for the Distribution Grid
}

\author{
Achilleas Tsitsimelis, Javier Matamoros and Carles Antón-Haro \\ Centre Tecnològic de Telecomunicacions de Catalunya (CTTC) \\ Parc Mediterrani de la Tecnologia - 08860 - Castelldefels - Barcelona (Spain) \\ email:\{achilleas.tsitsimelis, javier.matamoros, carles.anton\}@cttc.es
}

\begin{abstract}
In this paper, we propose a novel state estimation (SE) scheme for the distribution grid. It exclusively leverages on measurements from $\mu$ PMUs (micro Phasor Measurement Units) making it possible to operate at a faster time scale than conventional SE approaches based on legacy measurements. To circumvent observability issues, we realize that the voltage drop in adjacent buses is limited and, on that basis, we formulate a SE regularized weighted total variation estimation (WTVSE) problem. The problem can be iteratively solved by resorting to the so-called Alternating Direction Method of Multipliers (ADMM). Further, we provide closed-form expressions for the updates of the primal and dual variables. The performance of the proposed scheme is numerically assessed on a 95-bus distribution system for a number of realistic conditions of noise, load and photovoltaic generation profiles. A number of benchmarks are provided, as well.
\end{abstract}

\section{INTRODUCTION}

State Estimation (SE), namely, the determination of all the complex voltages in the power system from a set of measured variables from selected buses and branches, is a cornerstone for the monitoring of electricity grids. SE is a well-studied problem for the Transmission Grid (TG). In contrast, SE for the Distribution Grid (DG) has attracted lots of research interest over the last decade.

State estimation for DGs (unlike TGs) turns out to be very challenging. To start with, the limited availability of information from the distribution feeders as well as the specificities of DG network topologies often render the SE problem ill-posed. Concerning tree topologies, earlier works on DG SE [2], [3] have proposed alternative SE schemes suitable for radial-shaped DGs. As for the limited availability of measurements, substantial efforts have been devoted to strategies aimed to deal with the lack of system observability. Resorting to data mining techniques on daily load patterns, has been instrumental towards the definition of efficient strategies for the construction of pseudomeasurement sets [4]. By using such pseudomeasurements in conjunction with actual SCADA readings, (slightly) redundant measurement sets can be constructed. In [5] for instance, the authors investigate a two-time scale SE based on weighted least squares. Separating the information (SCADA, pseudomeasurements) according to their availability in time, the authors formulate the SE problem in a two-time scale fashion and investigate the efficiency of the resulting scheme under a diversity of load patterns. Furthermore, the increasing penetration of Distributed Energy Resources (DER) along with the introduction of new components, such as Electric Vehicles (EV) and distributed energy storage systems, have transformed the DG

This work was partially supported by the Catalan government under grant SGR2014-1567; the Spanish government under grant TEC2013-44591$P$ (INTENSYV), and the EU Seventh Framework Program under grants 607774 and 646469 (ADVANTAGE, P2P Smart Test).

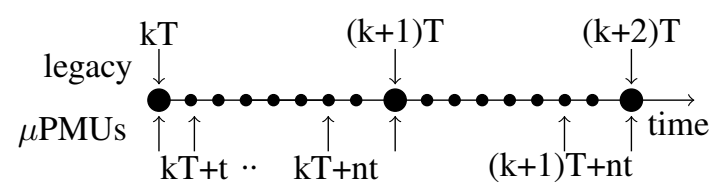

Fig. 1. Legacy and $\mu \mathrm{PMU}$ measurements are available at time instants $\{k T,(k+1) T, \ldots\}$ and $\{k T, k T+t, k T+2 t, \ldots\}$, respectively.

into an active infrastructure [1]. Besides, the power generation patterns of DERs (e.g., photovoltaic plants) exhibit variability at a shorter time scale. This results into an increasing number of voltage fluctuations and reverse power flows associated to the stochastic behavior of such DERs. Consequently, the interest in monitoring (and optimizing) DG's medium voltage systems with an increased temporal granularity has become a pressing need. To that aim, Distribution Management Systems (DMS) may resort to lowcost, high-resolution $\mu$ PMUs (micro Phasor Measurement Units) [7], [8]. Unlike legacy SCADA and pseudomeasurements, $\mu$ PMUs typically, operate at faster timescale (millisecond vs. second or minute timescales) and, in addition, the precision of their (linear and synchronized) measurements is higher.

In this paper, we propose a novel SE scheme for the distribution grid. The ultimate goal is to accurately estimate the system state at a faster time scale, that is, for the time instants between consecutive estimates based on legacy measurements (SCADA and pseudomeasurements) computed by conventional estimators based on normal equations. To that aim, we exclusively leverage on $\mu \mathrm{PMU}$ measurements (complex voltages) from a subset of buses. Due to operational constraints, the number of measurements available is unavoidably low, which compromises observability. To circumvent that, we resort to regularization techniques from the literature [10] leveraging on prior (expert) information on selected DG features. Specifically, we formulate SE as a weighted total variation estimation (WTVSE) problem which limits variations in voltage (state) estimates in adjacent buses. This stems from the fact that, in DGs, branch impedances (electrical lengths) and current flows tend to be lower than in TGs and so are voltage drops in adjacent buses [12]. On that basis, we propose a rule to define branchspecific weights for the regularizer. Then we effectively solve the constrained WTVSE problem by finding an iterative solution based on the so-called Alternating Direction Method of Multipliers (ADMM) [11] (unlike conventional gradient schemes based on normal equations). Closed-form expressions for the updates of the primal and dual variables are derived as well. 


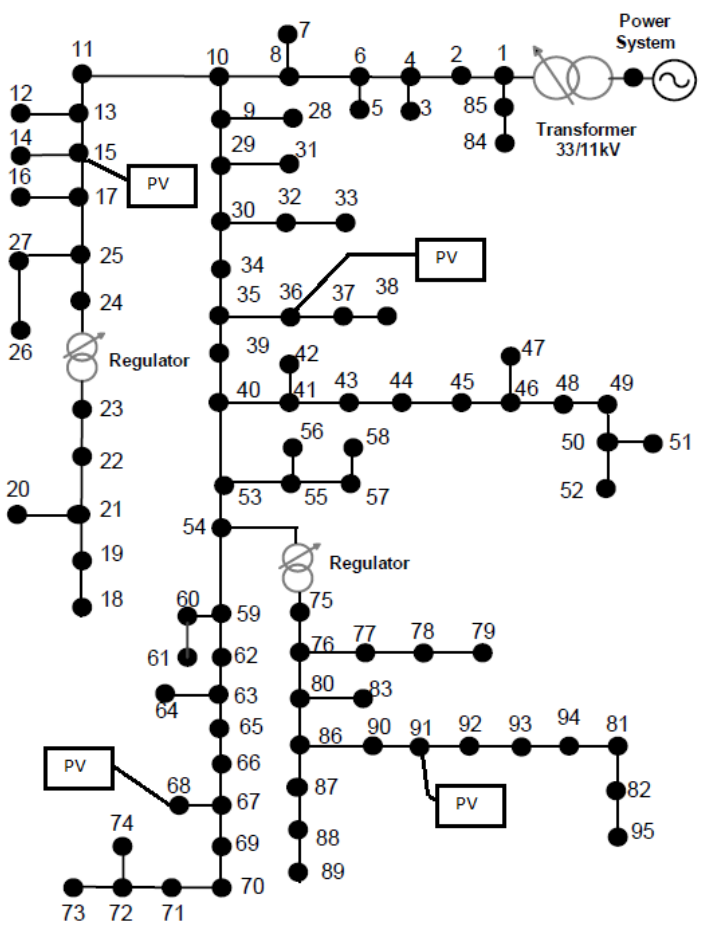

Fig. 2. A 95-bus radial distribution grid comprising four photovoltaic (PV) generation plants (adapted from [20]).

\section{System MODEL}

Consider a balanced radial distribution grid (see Fig.2) represented by the graph $\mathcal{G}=(\mathcal{V}, \mathcal{B})$, where $\mathcal{V}$ denotes the set of buses with cardinality $|\mathcal{V}|=N$; and $\mathcal{B}$ stands for the set of edges that describes their interconnections (branches), with cardinality $|\mathcal{B}|=N-1$. The complex current injections at the buses, i.e., $\mathbf{i}=\left[I_{1}, \ldots, I_{N}\right]^{T}$, satisfy $\mathbf{i}=\mathbf{Y} \mathbf{v}$, where $\mathbf{Y} \in \mathbb{C}^{N \times N}$ is the nodal admittance matrix $\left[15\right.$, Ch.7] and $\mathbf{v}=\left[V_{1}, V_{2}, . . V_{N}\right]^{T} \in \mathbb{C}^{N}$ stands for the complex voltages at all the buses that determines the state of the DG. The ultimate goal of power system state estimator (SE) is to estimate $\mathbf{v}$ from a set of measurements at selected buses and branches.

Two types of measurements are available in the aforementioned DG, namely, legacy and $\mu$ PMU measurements. As depicted in Fig. 1 , their collection is conducted at two different time scales and, moreover, observations are of different nature. The first category comprises readings from the SCADA system as well as pseudomeasurements. Legacy measurements are typically obtained at time intervals of $T=15$ minutes [5] and include: squared voltage magnitudes, $\left|V_{m}\right|^{2}$ for $m \in \mathcal{V}$; power flows at the branches, given by $S_{m, l}=P_{m, l}+j Q_{m, l}=V_{m} I_{m, l}^{*}$ for $(m, l) \in \mathcal{B}$; power injections, given by $S_{m}=P_{m}+j Q_{m}=V_{m} I_{m}^{*}$ for $m \in \mathcal{V}$ and squared branch current magnitudes, $\left|I_{m, l}\right|^{2}$ for $(m, l) \in \mathcal{B}$. In contrast, the sampling period of $\mu$ PMUs $t=T / q$ is smaller, typically on the order of few milliseconds [7], and provide the DG operator with updated snapshots of complex bus voltages, $V_{m}=\Re\left\{V_{m}\right\}+j \Im\left\{V_{m}\right\}$ for $m \in \mathcal{V}$. Notice that legacy measurements are non-linear functions of the system state, whereas $\mu$ PMU measurements turn out to be linear ones.

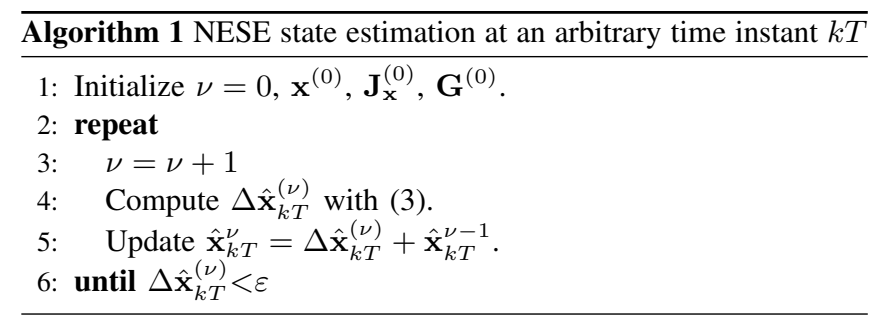

Let vector $\mathbf{x}=\left[\Re\left\{V_{1}\right\}, \Im\left\{V_{1}\right\}, \ldots, \Re\left\{V_{N}\right\}, \Im\left\{V_{N}\right\}\right]^{T} \in \mathbb{R}^{2 N}$ denote the Cartesian representation of the system state, and $\mathbf{z}=$ $\left[\mathbf{r}^{T}, \mathbf{y}^{T}\right]^{T} \in \mathbb{R}^{M}$ stand for the corresponding measurement vector, where $\mathbf{r} \in \mathbb{R}^{L}$ and $\mathbf{y} \in \mathbb{R}^{K}$ are the vectors of legacy and $\mu \mathrm{PMU}$ measurements, respectively. It is worth noting that, typically, we have $M=L+K>2 N$ but $K<2 N$ due to deployment and operational costs. Finally, we can write:

$$
\mathbf{z}=\mathbf{h}(\mathbf{x})+\mathbf{e}
$$

where $\mathbf{h}(\mathbf{x})$ denotes a non-linear function of $\mathbf{z}$ on $\mathbf{x}$ in compliance with the AC power flow model [15]; and $\mathbf{e} \in \mathbb{R}^{M}$ stands for zeromean Gaussian noise with known covariance matrix $\mathbf{R}_{\mathbf{e}}$.

\section{A Two-time scale State Estimation Scheme}

Bearing all the above in mind, we propose the following SE algorithm. At time instants $k T,(k+1) T, \ldots,(k+n) T$ for $k \in \mathbb{N}$, where both legacy and $\mu \mathrm{PMU}$ measurements are available, we adopt a conventional SE algorithm based on the Normal Equations (see Section III-A next). On the contrary, for the intermediate time instants between $k T$ and $(k+1) T$ we adopt a state estimator formulated as a constrained weighted total variation regularization problem (Section III-B). Note that the latter can only rely on the observations coming from the few $\mu$ PMUs available.

\section{A. Normal Equations-based State Estimator (NESE)}

According to (1), the conventional state estimator is given by the solution to the following non-convex optimization problem [6]:

$$
\hat{\mathbf{x}}=\arg \min _{\{\mathbf{x}\}}\|\mathbf{z}-\mathbf{h}(\mathbf{x})\|_{2}^{2}
$$

where, in the above expression, the time index has been dropped for the ease of notation. To solve (2), we resort to the so-called Normal Equations [11]:

$$
\mathbf{G}^{(\nu)} \Delta \mathbf{x}^{(\nu)}=\left[\mathbf{J}_{\mathbf{x}}^{(\nu)}\right]^{T} \mathbf{R}_{\mathbf{e}}^{-1}\left[\mathbf{z}-\mathbf{h}\left(\mathbf{x}^{(\nu)}\right)\right]
$$

where $\nu$ stands for the algorithm iteration index; matrix $\mathbf{J}_{\mathbf{x}}^{(\nu)} \in$ $\mathbb{R}^{M \times 2 N}$ denotes the Jacobian matrix of $\mathbf{h}(\mathbf{x})$ evaluated at $\nu^{t h}$ iteration (i.e., the matrix of all first order partial derivatives of the non-linear equations vector $\left.\mathbf{h}\left(\mathbf{x}^{(\nu)}\right)\right) ; \mathbf{G}^{(\nu)}=\mathbf{J}_{\mathbf{x}}^{(\nu) T} \mathbf{R}_{\mathbf{e}}^{-1} \mathbf{J}_{\mathbf{x}}^{(\nu)}$ stands for the gain matrix and $\Delta \mathbf{x}^{(\nu)}=\mathbf{x}^{(\nu+1)}-\mathbf{x}^{(\nu)}$ stands for the state vector difference between two consecutive iterations. Algorithm 1 summarizes the corresponding iterative procedure.

\section{B. Constrained Weighted Total Variation State Estimator (WTVSE)}

The following linear model relates the set of measurements provided by the $\mu$ PMUs at an arbitrary time instant $k T+n t$ (bus voltage phasors, in Cartesian coordinates) with the system state $\mathbf{x}$ (again, the time index has been omitted for the ease of notation):

$$
\mathbf{y}=\mathbf{H x}+\mathbf{w}
$$


where $\mathbf{H} \in \mathbb{R}^{K \times 2 N}$ stands for the corresponding measurement matrix [16], and $\mathbf{w}$ denotes zero-mean Gaussian noise with known covariance matrix $\mathbf{R}_{\mathrm{w}}$. In comparison with the full measurement vector $\mathbf{z}$, the number of measurements provided by the $\mu$ PMUs is typically lower than the dimension of the system state vector, i.e, $K \leq 2 N$. This renders the SE problem ill-posed since the system of equations (4) is underdetermined. This can be alleviated by introducing a regularization term accounting for the fact that, in DGs, the voltage difference between adjacent buses is small.

From all the above, the state estimation problem can be posed as a constrained weighted total variation regularization problem (WTVSE) [17]:

$$
\begin{gathered}
\hat{\mathbf{x}}=\arg \min _{\{\mathbf{x}\}} \frac{1}{2}\|\mathbf{y}-\mathbf{H} \mathbf{x}\|_{2}^{2}+\lambda\|\mathbf{W L} \mathbf{x}\|_{1} \\
\text { s.t. } \quad \tilde{\mathbf{Y}} \mathbf{x}=\mathbf{0}
\end{gathered}
$$

where, in the score function above, the term $\frac{1}{2}\|\mathbf{y}-\mathbf{H x}\|_{2}^{2}$ encourages fidelity in the solution to the vector of observations $\mathbf{y}$; $U(\mathbf{x})=\|\mathbf{W} \mathbf{L} \mathbf{x}\|_{1}$ is the regularization term, which introduces a prior model of the state vector $\mathbf{x}$; and $\lambda$ denotes the regularization parameter, which controls the tradeoff between data fidelity and prior knowledge. Matrix $\mathbf{L} \in \mathbb{R}^{(2 N-2) \times 2 N}$ is in charge of generating the vector with the voltage differences for each pair of adjacent buses $m$ and $n$. Since real and imaginary parts are stacked in the same vector, it can be expressed as

$$
\mathbf{L}=\mathbf{\Lambda} \odot \mathbf{I}_{2 \times 2}
$$

where, assuming that the elements in the $i$-th row of matrix $\boldsymbol{\Lambda} \in$ $\mathbb{R}^{N-1 \times N}$ are associated to the $(m, l)$ branch in the DG, they read

$$
[\boldsymbol{\Lambda}]_{i, j}= \begin{cases}1, & j=m \\ -1, & j=l \\ 0, & \text { otherwise }\end{cases}
$$

and, further, the operator $\odot$ denotes Kronecker product, and $\mathbf{I}_{2 \times 2}$ stands for the $2 \times 2$ identity matrix. The diagonal weighting matrix $\mathbf{W} \in \mathbb{R}^{(2 N-2) \times(2 N-2)}$ in the regularization term of problem (5) above can, in turn, be expressed as

$$
\mathbf{W}=\boldsymbol{\Omega} \odot \mathbf{I}_{2 \times 2}
$$

where (diagonal) matrix $\Omega \in \mathbb{R}^{(N-1) \times(N-1)}$ collects the weights for each branch. Taking into consideration that the voltage drop along the $(m, l)$ branch is proportional to the branch impedance $Z_{m, l}=R_{m, l}+j X_{m, l}$, that is, $V_{m}-V_{l}=Z_{m l} I_{m l}$ (see Fig. 3), a sensible choice for the aforementioned weights is

$$
\omega_{i, i}=1-\frac{|Z|_{m, l}}{|Z|_{\text {total }}}
$$

where we have assumed again that the $i$-th row of matrix $\Lambda$ is associated to the $(m, l)$ branch, and $Z_{\text {total }}$ denotes the total impedance for the feeder where the $(m, l)$ branch lies. By doing so, the larger the branch impedance, the smaller the weight, this allowing for larger variations in the corresponding buses. This allows to design a regularization term of (5) which properly reflects the specific characteristics (i.e., electrical lengths) of the various sections in medium-voltage feeders ${ }^{1}$.

${ }^{1}$ These may be substantially different in e.g., rural or urban areas.

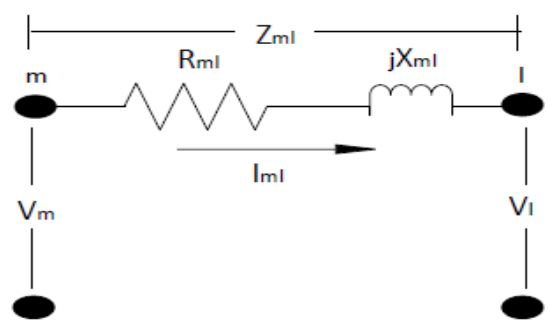

Fig. 3. Voltage drop along the $(m, l)$ branch.

Finally, the equality constraint $\tilde{\mathbf{Y}} \mathbf{x}=\mathbf{0}$ in problem (5) accounts for the $d$ zero current (power) injection buses. For instance, for the $m \in \mathcal{V}$ zero current injection bus, we have

$$
Y_{m m} V_{m}+\sum_{l \in \mathcal{N}} Y_{m l} V_{l}=0
$$

where $Y_{m m}$ stands for the sum of the admittances directly connected to bus $m, \mathcal{N}$ is the set of buses connected to the bus $m$, $Y_{m l}$ stands for the admittance that connect the buses $m, l$, and $V_{m}$ is the complex-valued voltage at bus $m$. By (i) splitting equation (10) into its real and imaginary parts; (ii) stacking the resulting two parts in consecutive equations of the system; (iii) leveraging on the Cartesian representation of the (complex) bus voltages in the system state vector $\mathbf{x}$; and (iv) re-arranging terms, the individual elements in matrix $\tilde{\mathbf{Y}}$ follow.

\section{Solving The WTVSE Problem VIA ADMM}

In order to solve problem (5), we resort to the Alternating Direction Method of Multipliers (ADMM) [10]. To that aim, however, we must re-write the problem as

$$
\begin{gathered}
\hat{\mathbf{x}}=\arg \min _{\{\mathbf{x}\}} \frac{1}{2}\|\mathbf{y}-\mathbf{H} \mathbf{x}\|_{2}^{2}+\lambda\|\boldsymbol{\theta}\|_{1} \\
\text { s.t. } \quad\left[\begin{array}{c}
\mathbf{W L} \\
\tilde{\mathbf{Y}}
\end{array}\right] \mathbf{x}-\left[\begin{array}{l}
\boldsymbol{\theta} \\
\mathbf{0}
\end{array}\right]=\mathbf{0}
\end{gathered}
$$

where $\boldsymbol{\theta}$ plays the role of an auxiliary variable and, hence, it introduces an additional constraint. Accordingly, the augmented Lagrangian reads:

$$
\begin{gathered}
\mathcal{L}(\mathbf{x}, \boldsymbol{\theta}, \boldsymbol{\mu}, \boldsymbol{\pi})=\frac{1}{2}\|\mathbf{y}-\mathbf{H} \mathbf{x}\|_{2}^{2}+\lambda\|\boldsymbol{\theta}\|_{1} \\
+\boldsymbol{\mu}^{T}(\mathbf{W L} \mathbf{x}-\boldsymbol{\theta})+\frac{c}{2}\|\mathbf{W L x}-\boldsymbol{\theta}\|_{2}^{2}+\boldsymbol{\pi}^{T}(\tilde{\mathbf{Y}} \mathbf{x})+\frac{c}{2}\|\tilde{\mathbf{Y}} \mathbf{x}\|_{2}^{2}
\end{gathered}
$$

with $\boldsymbol{\mu}$ and $\boldsymbol{\pi}$ standing for the vectors of Lagrange multipliers, and $c$ denoting a pre-defined positive constant. The ADMM iterates for the updates of the primal and dual variables become:

$$
\begin{aligned}
& \mathbf{x}^{\nu+1}=\arg \min _{\mathbf{x}} \mathcal{L}\left(\mathbf{x}, \boldsymbol{\theta}^{\nu}, \boldsymbol{\mu}^{\nu}, \boldsymbol{\pi}^{\nu}\right) \\
& \boldsymbol{\theta}^{\nu+1}=\arg \min _{\boldsymbol{\theta}} \mathcal{L}\left(\mathbf{x}^{\nu+1}, \boldsymbol{\theta}, \boldsymbol{\mu}^{\nu}, \boldsymbol{\pi}^{\nu}\right) \\
& \boldsymbol{\mu}^{\nu+1}=\boldsymbol{\mu}^{\nu}+c\left(\mathbf{W} \mathbf{L} \mathbf{x}^{\nu+1}-\boldsymbol{\theta}^{\nu+1}\right) \\
& \boldsymbol{\pi}^{\nu+1}=\boldsymbol{\pi}^{\nu}+c\left(\tilde{\mathbf{Y}} \mathbf{x}^{\nu+1}\right) .
\end{aligned}
$$




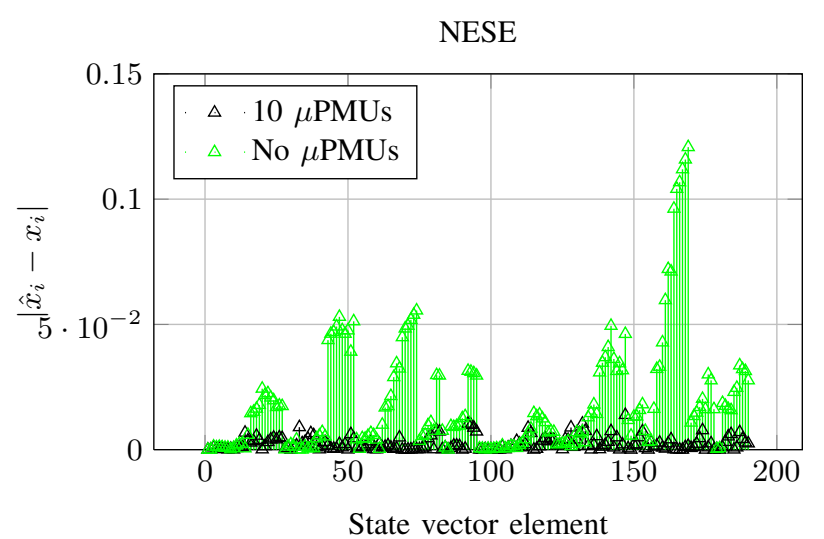

Fig. 4. Absolute error in the estimates of each element in the state vector (real and imaginary parts of voltages) with NESE.

One can easily prove that the closed form of the sequential updates of the primal variables at iteration $\nu$ can be written as

$$
\begin{gathered}
\mathbf{x}^{\nu+1}=\left(\mathbf{H}^{T} \mathbf{H}+c \mathbf{L}^{T} \mathbf{W}^{T} \mathbf{W} \mathbf{L}+c \tilde{\mathbf{Y}}{ }^{T} \tilde{\mathbf{Y}}\right)^{-1} \\
\left(\mathbf{H}^{T} \mathbf{y}-\mathbf{L} \mathbf{W}^{T} \boldsymbol{\mu}^{\nu}+c \mathbf{L}^{T} \mathbf{W}^{T} \boldsymbol{\theta}^{\nu}-\tilde{\mathbf{Y}} \boldsymbol{\pi}^{\nu}\right) \\
\boldsymbol{\theta}^{\nu+1}=\mathcal{S}_{\lambda / c}\left(\mathbf{W} \mathbf{L} \mathbf{x}^{\nu+1}+c^{-1} \boldsymbol{\mu}^{\nu}\right)
\end{gathered}
$$

with $S_{a}(\chi)$ standing for the soft thresholding operator, that is,

$$
S_{a}(\chi)= \begin{cases}\chi+a & \text { if } \chi<-a \\ 0 & \text { if }|\chi| \leq a \\ \chi-a & \text { if } \chi>a .\end{cases}
$$

\section{Numerical Results}

The performance of the proposed SE scheme has been assessed on the 95-bus distribution grid shown in Fig. 2 [20]. This DG, which is located in the UK, has a nominal voltage of $11 \mathrm{kV}$ and the installed active/reactive load is $3.83 \mathrm{MW} / 0.95 \mathrm{MVar}$, respectively. As Fig. 2 illustrates, we have included four photovoltaic (PV) plants. Their nominal capacity is $0.8,0.8,0.5$ and $0.4 \mathrm{MW}$ and they are connected to buses 15, 36, 68 and 91, respectively. Concerning observations, we have considered a set of legacy measurements with redundancy $L / N=1.4$. This includes (i) 5 voltage measurements located at the substation and the DER buses; (ii) 35 pairs of branch power flows $P_{i, j}, Q_{i, j}$; (iii) 8 squared current magnitudes at the feeders $\left|I_{i, j}\right|^{2}$; (iv) and 90 pairs of power injections $P_{i}, Q_{i}$, out of which 40 are pseudomeasurements, and 50 are zero power injection virtual measurements. The readings in the aforementioned measurement subsets are corrupted with i.i.d. Gaussian noise with standard deviations $(\sigma) 10^{-3}, 2 \cdot 10^{-3}$, $4 \cdot 10^{-3}, 1.5 \cdot 10^{-2}$, and $10^{-5}$, respectively. We have also included $10 \mu \mathrm{PMU}$ measurements from synchrophasors located at buses 1 , 50, 30, 70, 20, 80, 40, 90, 10, and 60, and with $\sigma=10^{-4}$. As for loads, they are assumed to be Gaussian-distributed with $P_{m} \sim \mathcal{N}\left(P_{m}, 0.06\right), Q_{m} \sim \mathcal{N}\left(Q_{m}, 0.06\right)$ for $m=1,2, . . N \in \mathcal{V}$. Unless otherwise stated, computer simulation results are averaged over 100 realizations of the noise, load and PV generation profiles. For the power flow analysis, we have used MATPOWER 5.1 [13]. Results have been verified through CVX [14].

First of all, we focus on the performance of the NESE scheme. To recall, it operates at time instants $k T$, when both legacy and

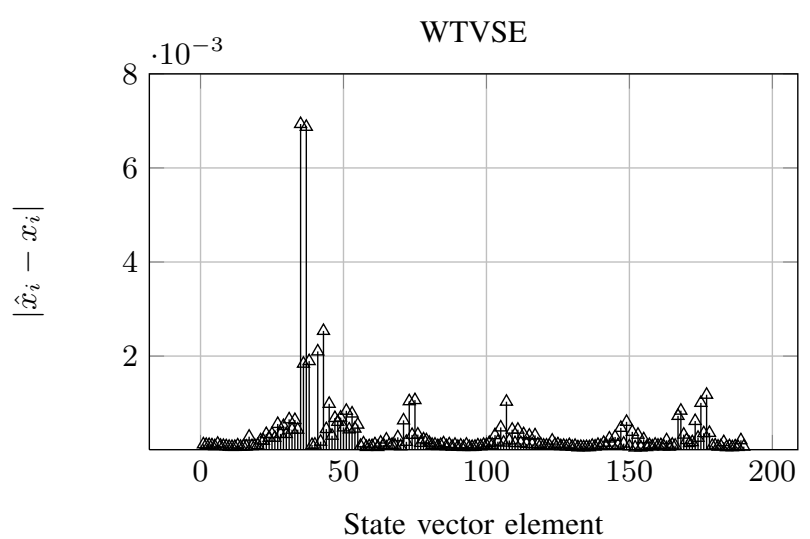

Fig. 5. Absolute error in the estimates for each element in the state vector (real and imaginary parts of voltages) with WTVSE $\left(\lambda=10^{-3}, c=1\right.$.)

$\mu \mathrm{PMU}$ measurements are available. Figure 4 shows the absolute error for each element of the state vector, i.e., $e_{i}=\left|\hat{x}_{i}-x_{i}\right|$ for $i=1, \ldots 2 N$. Clearly, the absolute error of the estimated voltages (both real and imaginary parts) decreases dramatically and stays below $1.5 \cdot 10^{-3}$ when (just) $K=20$ measurements (real and imaginary part of the complex voltages) from $10 \mu \mathrm{PMUs}$ are added to the set of $L=256$ legacy measurements. This gap is partly due the high percentage of pseudomeasurements $(100 / 256=39.1 \%)$ among legacy ones.

Next, we investigate the behavior of the proposed WTVSE scheme at the intermediate time instants, that is, between $k T$ and $(k+1) T$. Here, to recall, just $K=20$ observations from the subset of $\mu$ PMUs are available. Figure 5 reveals that, by introducing prior system information on the DG via the regularization term, WTVSE manages to achieve an accuracy comparable to that of the NESE system which requires the whole (legacy $+\mu$ PMUs) set of observations. This extent will be further elaborated later on. Finally, and as it could be anticipated, the largest errors tend to concentrate at the buses away from $\mu$ PMUs locations (e.g., feeder edges), see Fig. 2.

Complementarily, Fig. 6 illustrates the convergence behavior of the proposed WTVSE scheme for a varying number of $\mu$ PMUs. It is worth noting that such devices are incrementally introduced starting from the one at the primary distribution substation (bus number 1), up to bus number 60 (see details on actual locations in the first paragraph of this section). Unsurprisingly, the higher the number of $\mu \mathrm{PMUs}$, the lower the residual normalized error in the estimated state vector (yet the gain in excess of 8 synchrophasors is rather marginal). As for the number of iterations needed for the WTVSE scheme to convergence, it clearly depends on the number of available $\mu$ PMUs. Still, the number of iterations can be regarded as moderate (in the range of 3 to 8 ).

Further, we examine the impact of weights on performance. To that aim, we artificially increase the nominal values of the reactances (and, thus, impedances ${ }^{2}$ ) on branches 13-15, 14-15 and $15-17$ by a factor of 5 . The set of weights associated to the nominal and modified impedances are depicted in Fig.7 which reveals some changes in the aforementioned branches (whereas the rest of weights are quite similar, since so are branch impedances).

\footnotetext{
${ }^{2}$ This could e.g., account for longer lines in that geographical area
} 


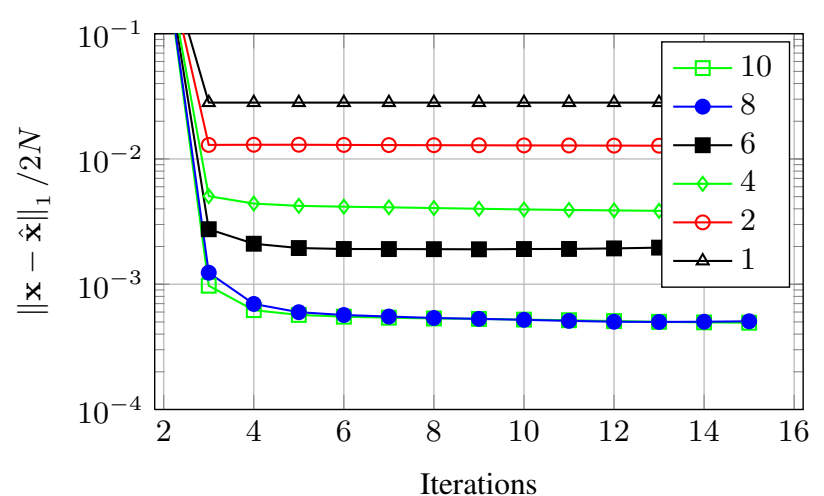

Fig. 6. Normalized error associated to the estimated state vector (1-norm) vs. iterations for a varying number of $\mu$ PMUs $\left(\lambda=10^{-3}, c=1\right.$.)

Besides, and in order to better illustrate the impact of voltage drop in the presence of high current levels, we have considered an industrial load of $1 \mathrm{MW}$ to bus 15 . Figure 8 depicts the absolute error in each element of the state estimate with nominal (top) and modified (bottom) impedances, and both in the presence of weights (WTVSE) or when all the weights are set to $\omega_{i, i}=1$ (TVSE). For the first scenario (nominal impedances), both algorithms provide comparable results since branch impedances are not that different. However, in the presence of dissimilar branch impedances, WTVSE clearly outperforms TVSE. This follows from the fact that WTVSE leverages on the prior knowledge on DG characteristics. And interestingly enough, this is despite of using a set of heuristic (yet sensible) weights.

Finally, we evaluate the ability of the proposed SE scheme to track short time-scale variations resulting from the use of PV energy resources. A sample of the aggregated power generation profile under consideration for the set of $4 \mathrm{PV}$ plants is depicted in Fig. 9 [18], [19], whereas the performance of the proposed SE scheme (along with that of two benchmarks) is shown in Fig. 10 next. In all cases, the estimation error is defined as the normalized absolute value of the difference between the actual state and the latest available estimate, computed by either the NESE or WTVSE schemes. Specifically, the 'NESE \& WTVSE' curve illustrates the estimation error associated to a SE running (i) the NESE scheme with both legacy and $\mu \mathrm{PMU}$ measurements at $k T$ time instants; and (ii) the proposed WTVSE scheme at the intermediate ones (we let $q=T / t=8$, for illustrative purposes). The 'NESE-last' curve provides a benchmark for the case where no SE scheme is used at intermediate time instants. Hence, we compute the estimation error between the actual system state at time $k T+n t$ and the latest available NESE estimate computed at $k T$ (on the basis of both legacy and $\mu$ PMU measurements). The 'NESE-updated PMUs' curve depicts the achievable error should NESE be run at intermediate time instants with the outdated legacy measurement collected at $k T$ (to preserve observability) and the updated $\mu \mathrm{PMU}$ readings gathered at $k T+n t$. Figure 10 reveals that, unsurprisingly, the estimation error for 'NESE-last' is the highest. This follows from the fact that both the legacy and $\mu \mathrm{PMU}$ measurements and, thus, the resulting state estimate are outdated. Indeed, the larger the deviation of the power generated by the PV plants at $k T+n t$ (see Fig. 9), the larger the estimation error (e.g. at $T+4 t$ ). By replacing

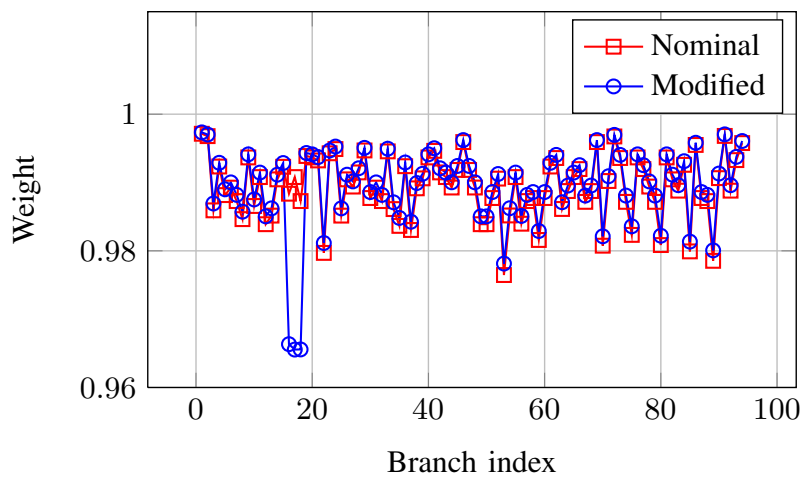

Fig. 7. Sets of weights for the scenarios with nominal and modified impedances.

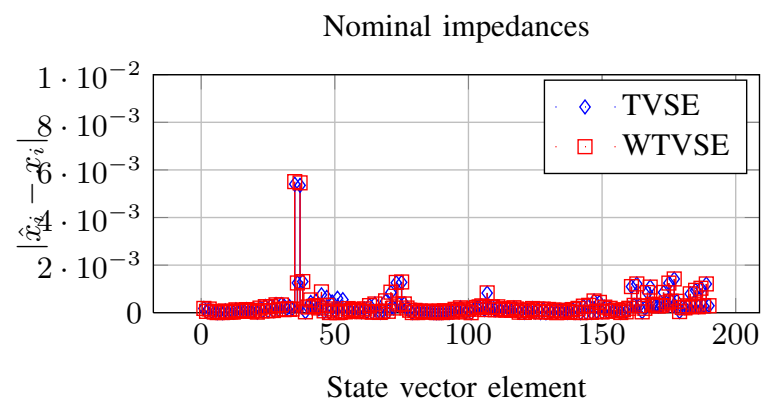

Modified impedances

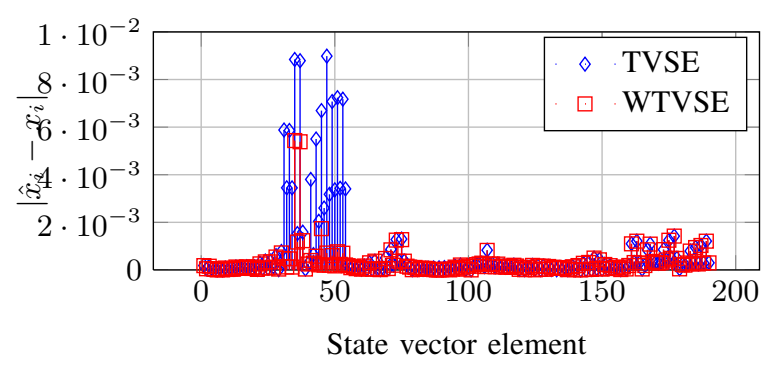

Fig. 8. Absolute error in the estimates for each element in the state vector (real and imaginary parts of voltages) with (W)TVSE for scenarios with nominal (top) and modified (bottom) impedances $\left(\lambda=1 \times 10^{-3}, c=1\right)$.

the outdated $\mu \mathrm{PMU}$ readings by updated ones ('NESE-updated PMUs' curve), performance substantially improves. This evidences the large impact that $\mu$ PMUs have on the computation of the system state. However, the proposed 'NESE \& WTVSE' scheme clearly outperforms both benchmarks. More importantly, for the simulated scenario, the estimation error in all intermediate time instants is even lower than that at $k T$ instants. In conclusion, by exploiting prior (expert) information on the DG via regularization (WTVSE) in conjunction with more sophisticated solvers (ADMM), we have obtained a SE scheme not requiring legacy measurements.

\section{CONCLUSIONS}

In this paper, we have proposed a regularized weighted total variation state estimation (WTVSE) scheme for the distribution grid. We have provided closed-form expressions for the updates of the primal and dual variables which are needed to iteratively solve the problem by means of the Alternating Direction Method 


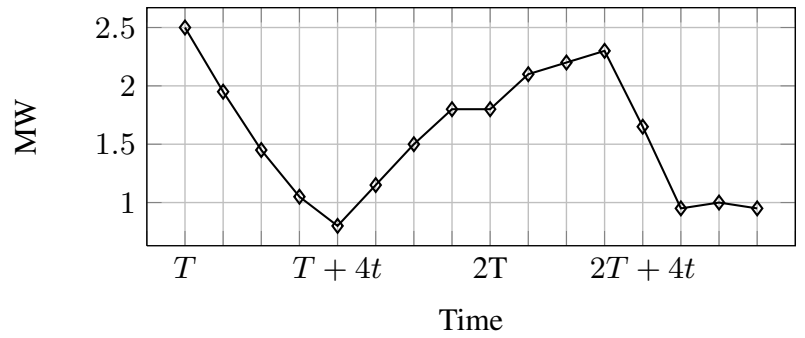

Fig. 9. Aggregated power generation profile for the four PV plants.

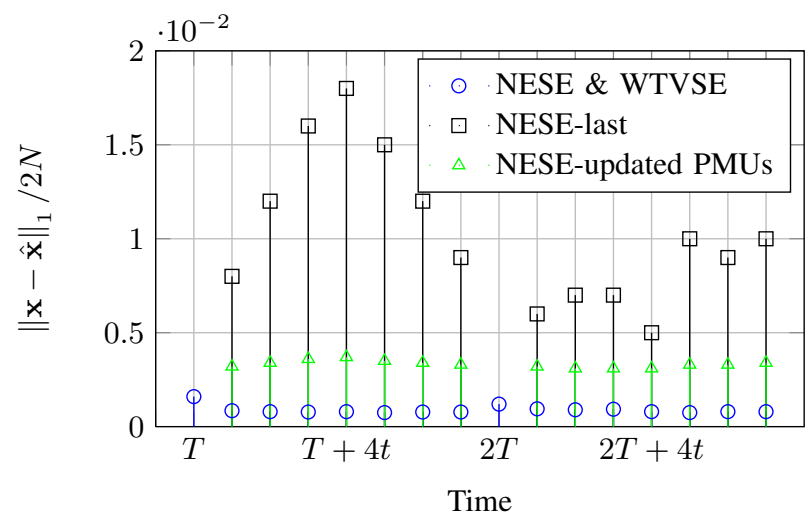

Fig. 10. Normalized error of the estimated state vector (1-norm) vs time $\left(\lambda=10^{-3}, c=1\right.$. $)$

of Multipliers (ADMM). We have also proposed a rule to compute the aforementioned weights as a function of branch and feeder impedances. Yet heuristic, the rule is sensible and particulary useful for DGs with dissimilar branches. Computer simulation results (on a 95-bus distribution system) indicate that (i) the introduction of prior information via a regularization term renders the SE problem solvable (observable); and (ii) WTVSE's estimation error is smaller than that of a conventional NESE scheme requiring the whole set of legacy and $\mu \mathrm{PMU}$ measurements. The latter holds for both the 'NESE-last' and 'NESE-updated PMU' benchmarks. The number of iterations needed for the WTVSE scheme to convergence is in the range of 3 to 8 which can be regarded as moderate. The higher the number of $\mu \mathrm{PMUs}$, the lower is the residual estimation error in the estimates.

\section{REFERENCES}

[1] A. Gomez-Exposito, A. Abur, A. de la Villa Jaen, and C. GomezQuiles, "A multilevel state estimation paradigm for smart grids", Proc. IEEE, vol.99, no.6, pp. 952-976, Jun. 2011.

[2] Whei-Min Lin and Jen-Hao Teng, "State estimation for distribution systems with zero-injection constraints," in IEEE Transactions on Power Systems, vol. 11, no. 1, pp. 518-524, Feb 1996.

[3] M. E. Baran and A. W. Kelley, "A branch-current-based state estimation method for distribution systems," in IEEE Transactions on Power Systems, vol. 10, no. 1, pp. 483-491, Feb 1995.

[4] E. Manitsas, R. Singh, B. C. Pal and G. Strbac, "Distribution System State Estimation Using an Artificial Neural Network Approach for Pseudo Measurement Modeling," in IEEE Transactions on Power Systems, vol. 27, no. 4, pp. 1888-1896, Nov. 2012.

[5] A. Gòmez-Expòsito, C. Gòmez-Quiles and I. Dżafic̀, "State Estimation in Two Time Scales for Smart Distribution Systems," in IEEE Transactions on Smart Grid, vol. 6, no. 1, pp. 421-430, Jan. 2015.
[6] R. Singh, B. C. Pal and R. A. Jabr, "Choice of estimator for distribution system state estimation," in IET Generation, Transmission \& Distribution, vol. 3, no. 7, pp. 666-678, July 2009.

[7] A. von Meier, D. Culler, A. McEachern and R. Arghandeh, "Microsynchrophasors for distribution systems," Innovative Smart Grid Technologies Conference (ISGT), 2014 IEEE PES, Washington, DC, 2014, pp. 1-5.

[8] Stewart, Emma M., et al. Using Micro-Synchrophasor Data for Advanced Distribution Grid Planning and Operations Analysis. No. LBNL-6866E. Ernest Orlando Lawrence Berkeley National Laboratory, Berkeley, CA (US), 2014.

[9] A. Abur, A. Gòmez-Expòsito, A. de la Villa Jaen, C. Gòmez-Quiles and P. Rousseaux, " On the Use of PMUs in Power System State Estimation", 17th Power Systems Computation Conference, 2011.

[10] S. Boyd, N. Parikh, E. Chu, B. Peleato, and J. Eckstein, 'Distributed optimization and statistical learning via the alternating direction method of multipliers", Found. Trends Mach. Learn., vol. 3, no. 1, pp. 1-122, 2010.

[11] S. Boyd and L. Vandenberghe, "Convex Optimization", Cambridge University Press, 2004.

[12] H. Ahmadi, J. R. Mart and A. von Meier, "A Linear Power Flow Formulation for Three-Phase Distribution Systems," in IEEE Transactions on Power Systems, vol. 31, no. 6, pp. 5012-5021, Nov. 2016.

[13] R. Zimmerman, C. Murillo-Snchez, and R. Thomas, "Matpower: Steady-state operations, planning, and analysis tools for power systems research and education", IEEE Trans. Power Syst., vol. 26, no. 1, pp. 12-19, Feb. 2011.

[14] Michael Grant and Stephen Boyd. CVX: Matlab software for disciplined convex programming, version 2.0 beta. http://cvxr.com/cvx, September 2013.

[15] J. J. Grainger, W. D. Stevenson, "Power System Analysis", Book, MacGraw-Hill, 1994.

[16] V. Kekatos and G. B. Giannakis, "Distributed Robust Power System State Estimation," in IEEE Transactions on Power Systems, vol. 28, no. 2, pp. 1617-1626, May 2013.

[17] Q. Yuan, L. Zhang and H. Shen, "Multiframe Super-Resolution Employing a Spatially Weighted Total Variation Model," in IEEE Transactions on Circuits and Systems for Video Technology, vol. 22, no. 3, pp. 379-392, March 2012.

[18] D. Cheng, B. A. Mather, R. Seguin, J. Hambrick and R. P. Broadwater, "Photovoltaic (PV) Impact Assessment for Very High Penetration Levels," in IEEE Journal of Photovoltaics, vol. 6, no. 1, pp. 295-300, Jan. 2016.

[19] R. van Haaren, M. M. Morjaria, and V. Fthenakis, "Empirical assesment of short term variability from utility-scale solar PV plants", Progress in Photovoltaics: Research and Applications, 2012.

[20] Yashodhan Prakash Agalgaonkar, "Control and Operation of Power Distribution System for Optimal Accommodation of PV Generation", Thesis of Doctor of Philosophy, Imperial College, Department of Electrical and Electronic Engineering, March 2014. 